\title{
Research on the Training Mode of Excellent Chinese Medicine Talents Based on the Background of Pilot College
}

\author{
Zheng Qiwei ${ }^{1}$, Wei Jianhong ${ }^{1}$, Zhou Yanping ${ }^{2}$, Shu Jinsong ${ }^{3}$, Cao Jigang ${ }^{1}$ \\ ${ }^{1}$ Dean's Office, Hubei University of Chinese Medicine, Wuhan, China \\ ${ }^{2}$ Clinical College of TCM, Hubei University of Chinese Medicine, Wuhan, China \\ ${ }^{3}$ Teacher Development Center, Hubei University of Chinese Medicine, Wuhan, China
}

Email address:

willzheng@foxmail.com (Zheng Qiwei),774941339@qq.com (Cao Jigang)

\section{To cite this article:}

Zheng Qiwei, Wei Jianhong, Zhou Yanping, Shu Jinsong, Cao Jigang. Research on the Training Mode of Excellent Chinese Medicine Talents Based on the Background of Pilot College. Higher Education Research. Vol. 3, No. 3, 2018, pp. 32-37.

doi: $10.11648 /$ j.her.20180303.11

Received: June 9, 2018; Accepted: July 7, 2018; Published: July 30, 2018

\begin{abstract}
The establishment of the pilot college is a comprehensive reform that takes the innovative talent training system as the core and the college as the basic implementation unit. Through literature review and experience summarization, this paper analyzes the training mode of Traditional Chinese Medicine (TCM) talents. And on the basis of this, the paper demonstrates the four aspects of restricting the training of TCM talents in current university education: the mechanism of talent selection, the mode of personnel training, the strength of teachers and the internal governance structure in Universities. In the end, this paper discusses some reforms in these four aspects, and hopes that through these reforms, we can reconstruct the training system of innovative talents in modern TCM education.
\end{abstract}

Keywords: Pilot College, Chinese Medicine, Talent Training Mode

\section{Introduction}

Traditional Chinese Medicine (TCM) is a fine traditional culture of the Chinese nation, a national treasure of the country, and a set specialized system with unique theoretical foundation that have been repeatedly practiced and verified by the working people of the country during the long struggle against disease. At present, TCM education has basically completed the transformation from traditional education model to modern scientific education model. At this stage, higher TCM education with institutions as the main carrier has become the main mode of cultivation of TCM personnel. However, after more than 50 years of development, TCM higher education has been plunged into new unfavorable development situations. The society has experienced problems such as the decrease of famous Chinese medicine doctors and the questioning of the quality of TCM talent training. As a result, the demand for famous TCM practitioners has become increasingly strong in society. Education is faced with various confusion and reform is urgently needed.

The pilot college is a comprehensive reform focusing on innovation talent training system, with colleges (departments, sections) as the basic implementation unit. In December 2012, the Ministry of Education issued the Guiding Opinions on Promoting the Reform of Pilot Colleges, which required the schools to reform in four aspects: student recruiting and selection methods, personnel training mode, teacher selection evaluation system, and college internal governance structure. And 24 supportive measures were provided subsequently [1]. This not only demonstrates the comprehensive reform of higher education, but also creates experience in the overall advancement of higher education system reform.

\section{Regularities of TCM Personnel Training}

\subsection{Study on the Regulations of Success of Ancient Chinese Medicine Experts}

Famous TCM doctors, namely, refer to TCM practitioners who are famous in the society, have good medical ethics and high reputation. 
Through analyzing regular patterns of famous Chinese medicine experts, such as Wang Hua and others [2], it's be found that the achievements of famous experts in traditional Chinese medicine have depended not only on their personal qualities and efforts, but also on the environment and education. For example, young medical students who pay attention to traditional culture, get familiar with Chinese medicine classics, combine reading with clinical evidence, collect and summarize more, are all strongly associated with the talents of traditional Chinese medicine experts.

\subsection{A Summary of the Successful Experience of Modern Chinese Medicine Experts}

Some study [3] analyzed the study motives, inheritance patterns, reading and study methods, clinical practice, and attitudes of scholars of a generation of old Chinese medicine doctors born in the late 19th and early 20th centuries, and summarized the experience of modern talents in modern Chinese medicine. According to them, the seven major factors of being an excellent TCM doctor are as follows: to strongly believe in Chinese medicine and establish their careers clearly; to learn from the teachers plus professional training; to read classics well and to read widely; to hold the constant clinical evidence and apply what they learn; to study classics to seek for the path; to think conscientiously and have the courage to innovate; to work hard and learn from others. The above factors constitute the basic framework for the success of modern famous doctors and it is the basic law that should be followed by those who wish to become masters of traditional Chinese medicine.

\subsection{Exploration of the Law of Higher Education on Modern TCM}

Chinese medicine has a history of thousands of years. While the history of the higher education of Chinese medicine under modern condition is comparatively short, and its laws are still in the process of development. For the law of higher education in Chinese medicine, the feasibility of its exploration depends on the two existing foundations: First, ancient Chinese medicine education with a long history provides a source for higher education of Chinese medicine under modern conditions; Second, after being explored for more than 50 years, the higher education in Chinese medicine has initially exhibited a certain degree of regularity, which makes it possible to explore the laws of higher education in Chinese medicine.

With the extension of TCM higher education practices and the development of the business, the regularity of the development have become increasingly visible. The space for the exploration of the laws of higher education in TCM has become broader, and its significance is even more farreaching. Compared with higher education, TCM higher education is a new thing, therefore, its development should certainly go through several stages. Therefore, it takes time to fully understand it. Under the existing conditions of society and disciplines, the understanding of the laws of higher education in ancient traditional disciplines is difficult to clarify in a short period of time. This hysteretic recognition will inevitably bring many problems to the higher education of Chinese medicine, especially to the major issues of cultivating a new generation of TCM talents. Therefore, it is imperative to study and explore the laws of higher education in Chinese medicine, whether it is for theories study or for the requirements of educational practice.

\section{The Restrictive Factors of Cultivating Talents of Traditional Chinese Medicine in Current College Education}

\subsection{Students Selection Mechanism}

The current selection mechanism of universities is based on the unified enrollment method for college admissions in the country, with "score selection" as the main form. In recent years, some colleges and universities have also conducted independent enrollment exams, some students who have a strong interest in Chinese medicine, and have certain basic knowledge of Chinese medicine have obtained the qualifications for priority admission [4]. However, as far as the widespread student population is concerned, the selection mechanism is still flawed. Whether or not students are suitable for the study of Chinese medicine cannot be determined by the results of only one examination. This method of "one test for profession" and "one test for life" has led to the loss of some outstanding students who are interested in practicing Chinese medicine in the future.

\subsection{Cultivation Mode}

At present, most Chinese medicine colleges have a relatively simple training mechanism. There are still some problems in the education concept, curriculum system setting, and clinical practice training.

1. In the actual training process, many educational concepts cannot be completed due to the objective immature conditions, and they become "empty and theoretical but not real", which also hinders the implementation of advanced educational concepts in teaching practice. For example, although the school began to vigorously promote the transformation from the traditional lecture-based teaching $\mathrm{LBL}$ to the $\mathrm{PBL}$ and CBL teaching methods, and some teachers gradually began to try, the full implementation of the new teaching model will still take a long time due to the special nature of the profession, the difference in the acceptance of students etc.

2. In the construction and improvement of the curriculum system, many colleges and universities have made certain adjustments. For example, the proportions of main courses and supplementary courses, and the coordination of professional courses and general education courses, etc. In TCM specialty courses, 
western medicine courses, humanity, Chinese national culture studies, and other related supplementary courses, adjustments are made in accordance with the requirements of students and social development requirements. However, this adjustment is not perfect, and it is also insufficient in frontier disciplines and cross-discipline learning and training. It cannot effectively promote the cultivation of innovative talents.

3. In terms of clinical practice training, on the whole, the clinical practicing time for students is relatively short, and their comprehension of clinical practice is shallow, which directly leads to the lower clinical practice ability, emergency response capacity, and processing capacity of most medical students. Of course, this is also related to the large number of students, the limited number of internship hospitals, and the heavy workload of doctors. To solve the existing problems in the cultivation of students' practical abilities, a series of tasks must be properly solved.

\subsection{The Quality of Faculty}

The strength of teachers is the key to improving the quality of teaching. As a University of Chinese medicine, the flow of talent is small and same-origin is strong because of the special nature of the profession. On one hand, this can ensure a more uniform educational concept and method, and can maintain the stability of the teacher's strength for a longer period of time. On the other hand, it can easily lead to the solidification of ideas, lack of awareness of innovation, and lagging communication with the outside world. At the current stage, teachers generally have high academic qualifications, but there are certain problems in how to allocate time and energy and coordinate teaching, research, and apprentice training.

\subsection{Governance of Colleges and Universities}

Sorting out the relationship among the colleges, affiliated hospitals, and universities has always been an important factor constraining the development of medical colleges and universities, it is also important for the reform of the medical college management system. If the three-party relationship cannot be reasonably interdependent, it will inevitably affect school education and clinical practice. At this stage, more medical colleges and universities began to adopt the "unification of college and hospital" model for management. This model has great advantages compared to former model, but regarding specific implementation, it still requires a series of supporting policies and will take a long period of time to sort out how to coordinate with the three parties, distribute and manage the responsibility, rights and benefits. [5]

\section{The Exploration of Excellent TCM Talents Training Model under the Background of Pilot College}

Based on the above analysis and research on the regularity and training mode of famous Chinese medicine practitioners, combined with the current status and development trends of college education the basic ideas of the reform of pilot colleges can be summarized as follows: to take updating the educational concept as the guide, innovative TCM talent training system as the core, focusing on the development and assessment of teachers and student groups, it will proceed from the three aspects of personnel training programs, curriculum system settings, and clinical practice to promote the comprehensive reform of pilot colleges. It mainly achieved breakthroughs in four aspects: recruitment and selection mechanism, innovative talent cultivation model, teacher management mechanism, and internal governance structure of the college, and actively explored the excellent TCM talent training model based on the background of the pilot college.

\subsection{Reform of Students Enrollment and Selection}

The pilot college is a special zone for reform and innovation in colleges and universities. It is suggested that the colleges and universities of Chinese medicine should be given a certain degree of autonomy in enrollment. Reform measures should include the real-name recommendation of secondary school principals, self-recommendation of candidates, and joint selection of provincial model high schools. For students who grew up in a traditional Chinese medicine family, have a long history of being influenced by traditional culture, and certain background and knowledge in traditional culture, or those who have the tendency of thinking in TCM syndrome differentiation, or have a keen interest in Chinese medicine disciplines and unique insights in Chinese medicine disciplines, recruitment policy can be appropriately flexible when enrolling students, which will help to improve the overall quality of Chinese medical practitioners and to ensure the cultivation of outstanding TCM talents.

\subsection{The Development Mode of Innovative Talents}

Based on the mode of higher TCM education, construct a traditional Chinese medicine development model which combines the tradition and modern education, synergize traditional culture education with humanistic education, and focus on three key points of training Chinese medicine personnel: the unification of educational idea \& educational practice, teaching system \& curriculum setting, clinical practice \& TCM thinking training.

\subsubsection{The Unification of Traditional Chinese Medicine Education Idea and Educational Practice}

In the long run, the university realizes that the advanced education idea is the basic precondition and guarantee of the university management and development, is the setting of the development goal and the development direction, and shows a kind of expectation to the school's future development state.

Nanjing University of Traditional Chinese medicine summed up over the years of Chinese Medicine Education 
concept, concise "humane, skill, humanity," the educational concept [6]; humane refers to a lofty ideals and noble character, benevolence refers to the feelings of fraternity and superb skills; Humanity refers to the unification and allround development of the ethics and skills. As the idea of university education in the 21 st century, it integrates tradition of TCM culture with modern education thought, combines the party's educational policy with the modern educator Cai Yuanpei's "personality standard" education idea, and lays the ideological foundation for training higher talents of Chinese medicine in the new century.

\subsubsection{The Unification of TCM Teaching System and Curriculum}

Traditional Chinese Medicine teaching emphasizes the core position of TCM culture and clinical practice ability in the cultivation of Chinese medicine practitioners. With "strong humanities, solid foundation, focusing on skills, improving higher quality" as the goal, medical students' "position competency" as the guidance, to integrate and optimize the four major education systems, namely, theoretical curriculum system, humanistic education system, practice curriculum System, scientific research innovation system. Through constant reform of teaching methods and means, constant adjustment and perfection of evaluation system, the 'thinking, skills and literacy' trinity of the position competency teaching system of Chinese medicine practitioners can be constructed. Curriculum is the basic connotation of the training mode, how to emphasize the training of the students' position competency in the course setting, is the key point of the reform. The curriculum of the pilot College adopts the form of "3 Platform +4 module"[7], 3 platform, namely public basic platform, Professional Foundation platform, professional course platform. 4 modules refer to the basic module, which refers to courses related to Chinese medicine. Chinese medicine is rooted in Chinese traditional culture, in addition to TCM theory, students should also have a certain degree of traditional culture, so as to fully understand and comprehend the profound connotation of the relevant theoretical system of TCM, as well as the essence of the Chinese medicine. Second, the main module, refers to the Chinese medicine curriculum, to set up a series of courses on traditional Chinese medicine, TCM diagnosis, Traditional Chinese medicine, herbal formulae, and the classical original works, such as Internal classics, Typhoid fever, Synopsis of golden Chamber, and Febrile diseases Treatise on febrile and miscellaneous diseases, to encourage everyone to "learn classics, recite classics and use Classics". Third, the supplementary module, refers to the Western medicine professional curriculum. This module follows the principle of "traditional Chinese medicine as the major, Western medicine as a supplement" to enrich the structure of professional knowledge. Four is the expansion module, refers to the new, the overlapping, the marginal discipline curriculum. It's purpose is to broaden the field of research and extend the idea of lifelong education into Chinese medicine education, and strengthen the education after graduation. This kind of curriculum set scientific and reasonable, not only embodies the differentiation of Chinese Medicine education, but also follows the organization law of modern higher education.

\subsubsection{The Unification of TCM Clinical Practice and TCM Thought Process Training}

The training of Chinese medicine talents must pay attention to the cultivation of clinical practice skills, only through early clinical, multiple clinical, recurrent clinical, can students grasp the corresponding clinical skills better, as much as possible accumulation of clinical experience, and constantly verify, consolidate and upgrade skills.

As the characteristic of TCM education, TCM thinking plays a leading role in the whole educational process, and how to strengthen the training of Chinese medicine thinking is also the key point of reform. Traditional Chinese medicine thinking can be trained from the following aspects:

1. In the course setting, the teaching order and proportion of Chinese and Western medicine courses can be reasonably adjusted, and TCM courses can be offered in stages. In the first stage, only Chinese medicine courses should be arranged, including basic courses and professional courses, which can reduce the learning of other knowledge caused by the proactive role, so that students learn the theory of traditional Chinese medicine without interference, while combining clinical learning with the teacher to establish good thinking of Chinese medicine; the second stage is to study the Western medicine course on the basis of TCM learning, Strengthen clinical practice skills through clinical and other methods. At the same time, it can also increase the Chinese traditional culture and other content of learning.

2. Clinical teacher in the daily teaching should adhere to the use of Chinese medicine, the law, prescription, medicine for dialectical treatment, try to prioritize TCM over western medication, apply only TCM if possible.

3. In the process of clinical training, students have to acknowledge the theory of teachers, but also to learn his methods. A strong clinical environment of Chinese Traditional medicine is conducive to the advantages of teacher-apprentice education. If students study in the pure Chinese medicine outpatient or ward, or are apprenticed to pure Chinese Medicine Tutors, this is considered to be the most ideal clinical environment.

4. Students are required to read the classics books including the four classics of traditional Chinese Medicine, strengthen the study of classic courses, and complete reading notes and learning experience.

5. In clinical teacher-apprentice education, through the teaching of repeated explanations and validation, students should master the treatment of teachers, prescriptions and other medical and pharmaceutical principles, after the outpatient, request to consult the relevant literature, independent thinking and summarize the learning of teacher-apprentice education, teaching 
teachers should carefully review the learning outcome, especially in the disease, medicine, to point out its deficiencies.

According to the student's summary, clinical teachers can find in time the knowledge of students, carry out teaching work to the different student, help students to build the thought process of Chinese medicine as early as possible, and enable the student to establish the confidence to learn Chinese medicine.

\subsection{Reform of Teacher Selection and Evaluation System}

The late president of Tsinghua University, Mei Yiqi, once said: "The so-called university, is not about how high the building is, but how extraordinary the master is." "Teachers are the main body of running schools, Without the active participation and strong support of teachers, it is impossible to guarantee the smooth progress of reform. University must adopt various ways to absorb everyone's wisdom and unite the consensus of reform."

\subsubsection{Enhance the Reform of Employment System, Optimize the Structure of the Teachers}

Through the reform of HR policy, to expand the autonomy of the pilot colleges to select teachers, encourage the pilot colleges to explore more suitable incentive policy to facilitate the characteristics of higher education and talent development rules, to acquire high-level talent and scarce talent. Implement the teacher appointment system. In accordance with the principle of "current policy for existing team, new approach for new talent", strengthen management and evaluation during employment term, carry out "promote and demote", "transfer position while not employed", explore more flexible and effective employment mechanism for new recruits, strengthen target assessment, carry out "fire while not-employed, "leave when failed to promote", to achieve the goal of optimizing teachers ' structure. In addition, by employing well-known experts to participate in the college's professional improvement and personnel training work, hiring a large number of excellent front line doctors of jointestablished hospitals to serve as part-time teachers, a special combination of "dual-teacher-type" teaching staff will be established [8].

\subsubsection{Implement the Classification Management of Teachers 'Positions, to Reform Teachers' Assessment Methods}

Carry out teachers ' position classification management, according to teaching-oriented, teaching and scientific research dual, scientific research-oriented, social service and development type, explore the qualification management system of teachers ' multi-channel career development, carry out comprehensive evaluation according to teaching, scientific research and service, implement "Grade level based upon the academic capability, income guaranteed by teaching quality. College should explore the establishment of annual salary system, set the orientation of the high quality and more pay, strengthen the incentive mechanism, arouse the enthusiasm of the teachers, and bring the teachers ' energy back to the talent development.

\subsubsection{Increase the Training of Teachers}

College should strengthen the training of the young and middle-aged teachers, give full play to the old professors and old experts in the classic course of traditional Chinese medicine, and rely on the teaching staff to carry out the research activities regularly; adhere to the "send out, invite in" principle, strengthen external cooperation and communication; Continuously improve the comprehensive quality of teachers.

\subsection{Adjusting the Internal Governance Structure of the College}

To abolish the basic administrative units of the trial college, the establishment of teaching responsibility Awarding system, try to transform from the administrative unit lead, to professor lead approach, enhance the dominant position of professors while teaching [9]. The leading professors are in charge of the development plan, curriculum, specialty construction, teaching reform, textbook selection and textbook construction, teaching evaluation, as well as the work of the Teaching Guidance Committee, the recruitment of professional teachers, assessment and teaching quality evaluation, and at the same time, on the basis of the establishment of college teachers responsible for different types of courses. Teaching responsibility professor to implement the annual salary system and tenure system [10].

\section{Conclusion}

In summary, a series of mechanism innovation is carried out from the aspects of Students ' enrollment and selection, talent training mode, teacher selection and evaluation system, college internal governance structure and so on, to realize the elite education of excellent Chinese medicine, and to form a solid foundation for the formation of the unique famous Doctor Circle of TCM talents Training mode, It is also the direction of further efforts to explore the famous TCM training model of TCM colleges and universities. In the future, college will emancipate the mind, boldly explore reform, according to the rules of Chinese Medicine Education and the essence of the success of famous doctors, break through the bottleneck of the cultivation of innovative talents, at the same time, through the reform of pilot colleges, to clarify ideas, methods and experience, for the development and reform of Chinese Medicine Higher Education.

\section{References}

[1] Directive opinion of the Ministry of Education on the reform of the pilot colleges [Z].

[2] Wang Hua, Liang Zhong, Liu Songlin, et. Study on the rules of the famous Chinese Medicine Scholars ' success in history [J]. 2013, 15 (2): 3-5. 
[3] Lu Chunzhong, Huang Bo, Zhan Wei, etc. Summary report on the successful experience of modern famous Chinese medicine practitioners [J]. 2011, (8): 703-709.

[4] Zheng Qiwei, Xiang Yuanxi, Shu Jingsong. Research on the rationalization of the preferential record policy for the major of Chinese Medicine. Journal of Nanjing University of Traditional Chinese Medicine [J]. 2017, (18):185-188.

[5] Wanke, Zeng Wei, Shu Jinsong, Zheng Qiwei, Liang Zhong. Current situation and Enlightenment of the management system of clinical teaching at home and abroad. Journal of Nanjing University of Chinese Medicine [J], 2014, 15 (03): 207-210.

[6] Wu Mianhua. humane, skill, humanity --- the educational idea and practice of Nanjing University of Traditional Chinese Medicine [J]. 2007, 8 (3): 173-176.
[7] Gao Baozhong, Zhai Shuangqing, Guo Jianzhen, et. Study on the training mode of Chinese Medicine practitioners ' colleges and universities [J]. 2007, 26 (5): 28-31.

[8] Zhuang huaifen. Intensify efforts to actively promote the comprehensive reform of the Pilot Academy [J]. Higher education in China, 2013 (22): 35-37.

[9] Zhang Liangyu. Research on reform of Pilot colleges [D]. Lanzhou: Lanzhou University, 2014.

[10] Li Jiajun. Find the right hand to promote the comprehensive reform of the Pilot Institute [J]. Higher education in China, 2013 (19): 12-15. 